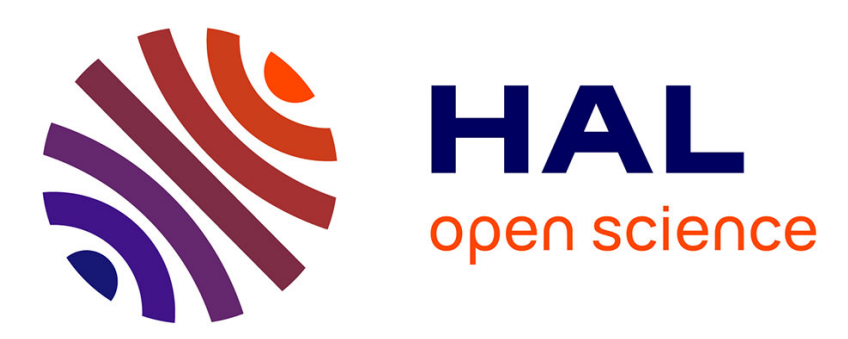

\title{
La notion d'autorité dans l'édition de la recherche : évolution des formes, changements d'autorité ?
}

\author{
Lise Verlaet, Alain Chante
}

\section{To cite this version:}

Lise Verlaet, Alain Chante. La notion d'autorité dans l'édition de la recherche : évolution des formes, changements d'autorité ?. Communication \& langages, 2017, 2017 (192), pp.27 - 46. 10.4074/S0336150017012030 . hal-01676344

\section{HAL Id: hal-01676344 \\ https://hal.science/hal-01676344}

Submitted on 5 Jan 2018

HAL is a multi-disciplinary open access archive for the deposit and dissemination of scientific research documents, whether they are published or not. The documents may come from teaching and research institutions in France or abroad, or from public or private research centers.
L'archive ouverte pluridisciplinaire HAL, est destinée au dépôt et à la diffusion de documents scientifiques de niveau recherche, publiés ou non, émanant des établissements d'enseignement et de recherche français ou étrangers, des laboratoires publics ou privés. 


\section{Communication \& langages}

http://www.necplus.eu/CML

Additional services for Communication \&

langages:

Email alerts: Click here

Subscriptions: $\underline{\text { Click here }}$

Commercial reprints: $\underline{\text { Click here }}$

Terms of use : $\underline{\text { Click here }}$

\section{La notion d'autorité dans l'édition de la recherche : évolution des formes, changements d'autorité ?}

Lise Verlaet et Alain Chante

Communication \& langages / Volume 2017 / Issue 192 / June 2017, pp 27 - 46

DOI: 10.4074/S0336150017012030, Published online: 01 November 2017

Link to this article: http://www.necplus.eu/abstract_S0336150017012030

How to cite this article:

Lise Verlaet et Alain Chante (2017). La notion d'autorité dans l'édition de la recherche : évolution des formes, changements d'autorité ?. Communication \& langages, 2017, pp 27-46 doi:10.4074/S0336150017012030

Request Permissions : $\underline{\text { Click here }}$ 


\section{La notion d'autorité dans l'édition}

\section{de la recherche :}

évolution des formes,

changements

d'autorité?

\section{LISE VERLAET \\ ET ALAIN CHANTE}

\section{INTRODUCTION}

Comme le rappelle Rieffel ${ }^{1}$, les historiens du livre distinguent quatre périodes clés dans l'évolution de la communication écrite, avec en premier lieu - de 4000 av. J.-C. à l'an 1000 - l'acquisition par l'homme de différents modes et supports d'écriture, lesquels ont abouti à une écriture alphabétique et l'utilisation du volumen. La seconde période - au $\mathrm{III}^{\mathrm{e}}$ siècle - est marquée par le passage du volumen au codex, soit une évolution du support d'écriture et ce faisant un bouleversement à la fois de la perception du texte et de son édition, laquelle devient quasi industrielle. En effet, à Rome, «l'industrie du livre [...] née sous la République, [s'est] développée sous l'Empire. [...] Les bibliopoles ${ }^{2}$ étaient installées au Forum, comme Atticus, ami de Cicéron, dans des boutiques avec affiches, où des esclaves tenaient le rôle de copistes, les Grecs étant les plus coûteux. Un lecteur dictait aux copistes réunis dans une salle, et un nombre considérable d'exemplaires étaient transcrits simultanément, cent exemplaires à l'heure du deuxième livre des Épigrammes de Martial, par exemple $»^{3}$.

1. Rémy Rieffel, Révolution numérique, révolution culturelle ?, Gallimard, 2014.

2. Le bibliopola (de biblion livre et polein vendre) doit être distingué du librarius : les bibliopoles étaient les éditeurs, tandis que les libraires étaient au service des particuliers

3. Antoine Compagnon, "Qu'est-ce qu'un auteur ? Introduction : mort et résurrection de l'auteur ", cours de licence, université Paris-Sorbonne, http://www.fabula.org/compagnon/auteur1.php

Cet article interroge la notion d'autorité scientifique à travers l'étude de l'évolution des formes de communications inhérentes à l'édition de la recherche et soulève, ce faisant, l'influence de ces dernières sur les changements d'autorité. La question de l'autorité est ainsi analysée dans sa complexité communicationnelle : qu'il s'agisse des origines étymologiques et historiques de la notion, des débats qu'elle suscite entre éditeurs, auteurs et militants, ou du rôle que les dispositifs d'u écrilecture " jouent dans l'organisation et publicisation des connaissances scientifiques contemporaines.

Mots clés : autorité scientifique, édition de la recherche, revue scientifique, évolution 
L'invention de l'imprimerie par Gutenberg en 1450 est considérée comme la troisième période. Des maisons d'édition se développent, porteuses de nouvelles exigences inhérentes aux risques économiques et financiers (investissement coûteux, tirages et stocks importants, prix bas) et à la recherche de la sécurité (concurrence et risque de plagiats). Elles demandent donc des protections sous forme de garanties et privilèges, un nouvel état de droit ${ }^{4}$, auquel l'État y répondra d'autant plus que l'accroissement des livres et des lecteurs favorise l'émergence d'idées nouvelles, ce qui provoque une attention et une autorité accrues de l'État, qui trouvera plus de moyens légaux pour intervenir sur la maison d'édition que sur l'auteur lui-même. Dans le même temps, les relations entre auteurs se modifient, ils doivent déterminer des stratégies complexes, entre la possibilité de publier davantage et une exposition au jugement et au plagiat. Nous vivons actuellement la quatrième période, celle de la communication électronique et des écrits d'écran ${ }^{5}$. Ces millénaires d'écriture et ces siècles d'imprimerie ont permis de diffuser et d'organiser les connaissances humaines, parallèlement se sont instituées une structure et une hiérarchie des pouvoirs entre l'auteur, l'éditeur (voire l'imprimeur) et le distributeur, avec l'intervention de médiateurs (tels que le critique et le bibliothécaire) et de modes d'évaluation. Or en à peine deux décennies, Internet a bouleversé notre rapport à l'information et à la communication, les écrits d'écran influent sur nos habitudes de lecture, d'écriture ${ }^{6}$ et imposent ainsi - à l'instar des précédents progrès techniques - des médiations nouvelles et de nouveaux rapports de force à la fois dans la structuration du savoir et dans le monde de l'édition.

$\mathrm{Au}$ sein de cet article, nous nous intéresserons à la notion d'autorité dans le secteur de l'édition scientifique, et plus particulièrement de l'édition de la recherche à travers l'étude des périodiques scientifiques. Pour ce faire, nous prendrons appui sur des recherches documentaires, l'observation et l'analyse de l'évolution des formes et des pratiques inhérentes aux revues scientifiques. Ainsi, après avoir interrogé les différentes facettes de la notion d'autorité, nous soulignerons l'influence liée à l'évolution des supports et techniques de communication scientifique sur les détenteurs de l'autorité. Subséquemment, nous rappellerons comment l'autorité des éditeurs commerciaux a dérivé vers un système oligarchique dont les rouages finissent par nuire à la recherche et aux sciences. Nous verrons également comment l'espace numérique a créé un appel d'air, lequel permet aux acteurs scientifiques de réinvestir le secteur de l'édition de la recherche et de reconquérir l'autorité dont ils avaient été destitués. Nous

4. Ibid.

5. Emmanuël Souchier, "L'écrit d'écran, pratiques d'écriture \& informatique », Communication \& langages, 107, 1996, p. 105-119.

6. Jean Clément, « Du livre au texte Les implications intellectuelles de l'édition électronique », Revue Sciences et techniques éducatives, 5(4), 1998, p. 401-409, <edutice-00135808>. 
soulignerons enfin les différents outils d'écrilecture ${ }^{7}$ augmentée pour le $\mathrm{Web}^{8}$, qui participent à cette réappropriation et - de notre point de vue - la font évoluer.

\section{LA NOTION D'AUTORITÉ, UNE NOTION POLYSÉMIOUE}

\subsection{L'autorité}

La notion d'autorité peut s'aborder à plusieurs niveaux. L'autorité est une forme de "domination fondée sur la légitimité de celui qui la possède »" ${ }^{9}$, toujours liée à un ensemble de conditions et de représentations variables dans le temps et dans l'espace ${ }^{10}$, qui ne relève " ni de la force ni de la persuasion ${ }^{11}$. Comme l'écrit François Bourricaud ${ }^{12}$, « l'autorité, c'est la personnalisation des règles, leur incarnation, ou encore la transfiguration symbolique de certains individus qui prennent à leur charge les normes collectives ». Ce " pouvoir d'agir sur autrui » peut prendre une dimension légale en vue du gouvernement des personnes ou des choses ou se placer au niveau d'une influence d'ordre intellectuel, moral, psychique permettant de s'imposer comme valeur, référence dans l'opinion publique (définition du Trésor de la Langue française).

$\mathrm{Au}$ niveau général, l'autorité, sa crise, sa disparition ou sa restauration nécessaire remplissent les discours politiques, journalistiques, pédagogiques. Ainsi, l'autorité est très discutée en pédagogie, s'avérant, en fait, un problème permanent pour les éducateurs. "Pourquoi en est-il ainsi ? Tout simplement parce que la relation d'autorité exclut la construction d'un véritable rapport à l'autre et fuit la question du "vivre ensemble" [...] parce que l'autorité est un "coup de force initial" et n'est, fondamentalement, que cela. " ${ }^{13}$ Houssaye affirme ainsi que l'autorité est incapable de justifier et de fonder la loi : «À proprement parler, quand elle n'est pas nuisible, l'autorité est inutile [...] Tant et si bien qu'à l'école, entre autorité et éducation, il faut choisir ${ }^{14}$. À l'inverse, elle est jugée hautement

7. Néologisme fondé pour exprimer la tendance face au texte numérisé à le manipuler, l'interroger, l'amender, contrairement à la quasi-sacralité du texte écrit traditionnel, jugé intangible. Par ses caractéristiques physiques mêmes, un écran pour lire et un clavier pour écrire, l'ordinateur pousse le lecteur à exercer une action concrète sur le texte. Il s'agit non plus seulement de lire, mais aussi d'écrire. C'est ce qui fait dire à Alain Vuillemin, qui reprend le néologisme au médiéviste canadien Serge Lusignan, qu'il n'est plus question dans ces dispositifs de lecture à proprement parler mais d'écrilecture. ("La lecture interactive et l'écriture », in Alain Vuillemin \& Michel Lenoble (dir.), Littérature, informatique, lecture, Limoges, Presses universitaires de Limoges, 1999, p. 101-110).

8. Évelyne Broudoux, «Contours du document numérique connecté » Documents et dispositifs à l'ère post-numérique: CIDE18, Édition Europia, 2015, p. 7-15. Évelyne Broudoux, «L'écosystème scientifique à l'heure de la participation sur le Web ", in Yves Jeanneret et al., Pratiques et usages numériques : H2PTM'13, Hermès-Lavoisier, 2013, p. 239-256.

9. Max Weber, Économie et société [1922], Paris, Pocket, 2003.

10. Alexandre Kojève, La notion de l'autorité [1942], Paris, Gallimard, 2004.

11. Pierre Karila-Cohen, «L'autorité, objet d'histoire sociale » Le Mouvement Social, 3, 2008, p. 3-8.

12. François Bourricaud, Esquisse d'une théorie de l'autorité, Paris, Plon, 1969.

13. Jean Houssaye, Autorité ou éducation : Entre savoir et socialisation : le sens de l'éducation, ESF, 2007.

14. Ibid., 2007. 
nécessaire au niveau scientifique, où elle intéresse de nombreuses disciplines : philosophie $^{15}$, psychologie sociale ${ }^{16}$, sociologie ${ }^{17}$ et droit ${ }^{18}$. Elle prend alors plusieurs aspects. Parmi les critères qui fondent l'autorité se trouvent l'authenticité et la valeur, c'est-à-dire la garantie de conformité qui donne une valeur scientifique. Puis la notoriété, la renommée, source de pouvoir.

En science de l'information, une autorité (ou notice d'autorité) sert à identifier sans ambiguïté des personnes, des choses ou des concepts. La forme retenue fait autorité, d'où son nom, calqué par ailleurs sur l'expression anglaise d'origine, authority control. On obtient ainsi en bibliothéconomie des listes des termes normalisés qui doivent être obligatoirement et nécessairement utilisés dans l'indexation. On est là au niveau de la donnée et d'un aspect éminemment formel, dans une normalisation imposée par des médiateurs (des bibliothécaires) pour la facilité de leur travail. Normalisation qui va s'imposer aux chercheurs pour la présentation de leurs ouvres et de leurs bibliographies comme preuve de l'authenticité de leurs sources et de la fiabilité de leur démarche.

\subsection{Autorité et auteur}

Il y a normalisation de la référence, car « le nom d'auteur est indispensable à toute classification bibliographique : il désigne une ouvre comme une étiquette sur un bocal ${ }^{19}$, mais " investies d'une fonction mémorielle [...] ces listes sont aussi le témoignage concret de la production intellectuelle ${ }^{20}$. Par ces listes, le nom d'auteur « assure une fonction classificatoire : il permet de regrouper des textes [...] de les rapprocher, de les authentifier, de les expliquer mutuellement [...] Le texte à auteur [...] est destiné à survivre dans le monde des textes ${ }^{21}$. Celui qui établit la liste et certifie l'authenticité « pose un geste de contrôle sur ce qui est perçu, conçu, observé et défini » ${ }^{22}$. Il s'agit là d' "un acte de représentation et un acte de définition, soit des actes de pouvoir [... .] et de structuration des réalités ${ }^{23}$. L'auteur a de l'autorité, est une autorité.

15. Voir notamment : Chantal Delsol, L'autorité, Paris, PUF, 1994. Alexandre Kojève, La notion de l'autorité, Paris, Gallimard, 2004. Myriam Revault d'Allonnes, Le pouvoir des commencements. Essai sur l'autorité, Paris, Seuil, 2006.

16. Richard Sennett, Autorité, Paris, Fayard, 1980.

17. François Bourricaud, Esquisse d'une théorie de l'autorité, Paris, Plon, 1969.

18. Antoine Garapon, Sylvie Perdriolle, Quelle autorité ? : une figure à géométrie variable, Autrement, 2000.

19. Antoine Compagnon, «Qu'est-ce qu'un auteur ? Introduction : mort et résurrection de l'auteur », op. cit.

20. Viviane Couzinet, "Fabrique de la liste : un dispositif entre mémoire et commémoration ", $2^{\mathrm{a}}$ Jordana Cientifica Internacional Redes e Processos Info-Communicacionais : Mediaçiões, Memoras, Apropriações, Rio de Janeiro, du 24 au 26 octobre 2012, p. 132-151.

21. Michel Foucault, "Qu'est-ce qu'un auteur ? », texte d'une conférence donnée en février 1969 à la Société française de philosophie, Dits et écrits, Gallimard, 1994.

22. Gilles Willett (dir.), La communication modélisée, Ottawa, Édition Erpi, 1992.

23. Alain Chante, «La notion de catalogue : de l'imprimé au numérique », Culture \& musées, 21, 2013, p. 131-152. 
Il ne faut pas croire que celle-ci ait toujours été évidente, tout comme la nécessité de l'indication de l'auteur, fluctuante. Ainsi, les textes « littéraires » ont été longtemps reçus sans nom d'auteur et dans l'anonymat de leur énonciation, leur ancienneté étant une autorité suffisante, alors que les textes scientifiques portaient un nom d'auteur, garant de leur autorité et signe de leur approbation. Puis cela s'est inversé, à partir des $\mathrm{XVII}^{\mathrm{e}}$ et $\mathrm{XVIII}^{\mathrm{e}}$ siècles, un anonymat croissant a caractérisé les textes scientifiques, jouissant de l'autorité de la science, tandis que le discours littéraire a dû être attribué ${ }^{24}$. L'on pourrait à ce propos paraphraser Barthes ${ }^{25}$ : " c'est la science qui parle, ce n'est pas l'auteur ». C'est que le texte scientifique a vocation à échapper à son contexte d'origine, à continuer à être lu et utilisé après lui, hors de lui. Mais que devient l'auteur ? Un simple initiateur, créateur d'une chose qui tombe dans le domaine public et ne lui appartient plus ${ }^{26}$ ? ou un inventeur qui garde des droits ? La connaissance se distinguait de l'invention en ce qu'elle ne pouvait pas être brevetée. Depuis Platon, le savant se voulait dans un autre univers que le commerçant. Et la théorie économique néoclassique a défini les droits de propriété intellectuelle en accordant à l'inventeur un monopole partiel et temporaire pour stimuler l'innovation et l'investissement en corrigeant donc cette défaillance du marché. C'est le cas des brevets, mais pour les articles, on reste dans le don maussien, l'auteur donnant son texte en contrepartie de la renommée qu'il retirera de sa publication.

\subsection{Actor et auctor}

Il y a deux origines latines assez proches du mot « auteur », qui se contaminent. Le dictionnaire Gaffiot indique :

- Actor (du verbe ago mettre en mouvement, pousser à, faire, être actif, viser à, exprimer par la parole) : celui qui fait mouvoir, avancer, celui qui fait et qui est donc un acteur mais aussi un auteur. On pourrait traduire par une expression contemporaine péjorative : " un faiseur »; celui qui fait, sans plus, sans génie, sans grande personnalité, sans autorité manifeste.

- Auct (du verbe augeo faire croître, augmenter, grandir, grossir par la parole) : celui qui augmente, qui fait progresser, celui qui confirme, le garant, la source d'autorité (écrit au Moyen Âge « auctorité »), l'auteur qui fait autorité, qui est respecté et cru. Le dérivé auctoritas fait de l'auteur « celui qui par son œuvre détient l'autorité ». Toute personne qui écrit ou a écrit n'est pas un auteur, la différence étant la même que celle entre le document et le monument ${ }^{27}$. Seul le rédacteur

24. Antoine Compagnon citant Michel Foucault, «Qu'est-ce qu'un auteur ? Introduction : mort et résurrection de l'auteur", op. cit.

25. La phrase d'origine est «C'est le langage qui parle, ce n'est pas l'auteur » (Roland Barthes, «La mort de l'auteur» (1968), Le Bruissement de la langue, Seuil, 1984).

26. Ceci pose notamment le problème de la notion de validité. Cette dernière peut-elle être maintenue? Quel(s) critère(s) permet(tent) de séparer une interprétation valide d'une mésinterprétation ?

27. Sur les relations document/monument, voir Patrick Fraysse, Le patrimoine monumental en images : des méditations informationnelles à la conversion monumentaire des documents, thèse de doctorat en SIC, université Toulouse 2, 2006 ; Patrick Fraysse, Gérard Régimbeau, « Le patrimoine architectural entre monuments-phares et documents monumentaires. La transmission des connaissances, des savoirs et des cultures : Alexandrie, métaphore de la francophonie ", Actes du Troisième colloque international $d u$ CIDEF (Centre international de documentation et d'échanges de la francophonie - Québec) - AFI 
dont les écrits sont reconnus comme des monuments par l'institution littéraire (ou scientifique) atteint l'autorité de l'auteur. Cette idée de reconnaissance est importante : l'auteur fait autorité parce qu'on lui reconnaît cette autorité par des démarches « info-documentaires ", depuis le Moyen Âge, où " pour être vraiment auteur (auctor), il faut que le nom soit cité, que les œuvres soient l'objet de copies nombreuses et de commentaires et qu'elles soient citées sous forme d'extraits. C'est à ce prix qu'il y a alors une vraie auctoritas ${ }^{28}$. De là vient l'autorité du médiateur, dont le pouvoir d'inscrire dans la norme va donner un gage de sérieux et symboliser l'autorité scientifique dont ont besoin le texte et l'auteur. On peut prendre des exemples très actuels : ce qui importe, ce n'est pas d'écrire un livre, c'est de trouver qui l'éditera ; ce n'est pas d'avoir « sorti » un livre, c'est que ce livre soit cité dans les bases de données.

\subsection{Auteur et auctorialité}

Il est alors question d'un problème de communication et d'image. L'autorité, prise au sens de la « valeur » interne de la connaissance contenue dans l'œuvre, n'est plus suffisante, elle se décale vers une autorité donnée de l'extérieur, une reconnaissance. On est là dans la "fonction auteur ", ou authorship, dirait-on en anglais, terme parfois traduit par le néologisme " auctorialité », pour justement le distinguer de l'autre dérivé étymologique : " autorité ». Ainsi, Foucault s'attache aux traits qui font l'auctorialité d'un discours plus que son autorité ${ }^{29}$, ce qui consisterait à interroger la définition ou l'image de l'auteur mise en jeu à partir de contrats tacites de communication.

La « clause auteur » est proposée aux lecteurs potentiels par un écrivant qui " parie » sur la coopération de ses interlocuteurs. . . Reste alors à savoir qui est celui qui articule le contrat littéraire, l'instance qui revendique une conception de l'auteur ou une image spécifique ${ }^{30}$. En effet, trois « lecteurs » successifs sont à prendre en compte : l'éditeur potentiel qui sélectionne au nom de critères scientifiques et au nom de critères commerciaux, les médiateurs, parmi lesquels on pourrait compter le journaliste et le bibliothécaire, comme le fait Dominique Wolton $^{31}$, et le public lui-même.

\section{L'INSTITUTIONNALISATION DE L'AUTORITÉ DANS LE SECTEUR DE L'ÉDITION SCIENTIFIQUE}

Si certaines pratiques de production de textes savants étaient déjà à l'œuvre au XIII ${ }^{\mathrm{e}}$ siècle ${ }^{32}$, il est communément admis que l'édition scientifique débute

(Agora francophone internationale - Paris), Alexandrie (Égypte), Bibliothèque d'Alexandrie, du 12 au 15 mars 2006, http://hal.archives-ouvertes.fr/hal-00607126/ [12 p.].

28. Françoise Vielliard, "Auteur et autorité dans la littérature occitane non lyrique », Auctor et auctoritas: Invention et conformisme dans l'écriture médiévale, École des chartes, 2001, p. 375-389.

29. Michel Foucault, "Qu'est-ce qu'un auteur?», op. cit.

30. Melliandro Mendes Gallinari, "La "clause auteur" : l'écrivain, l'ethos et le discours littéraire ", Argumentation et Analyse du Discours, 3, 2009, http://aad.revues.org/663

31. Dominique Wolton a rappelé que « journalistes et bibliothécaires doivent reprendre les rênes de l'information ", propos évoqués par Cécile Touitou, "Les nouveaux usages des générations internet ", $B B F, 4$, juillet 2008, p. 67-70, <http://bbf.enssib.fr/consulter/bbf-2008-04-0067-001>.

32. Guylaine Beaudry, La communication scientifique et le numérique, Paris, Hermès Lavoisier, 2011. 
dès la création de l'imprimerie avec la possibilité pour les savants de se référer à des supports écrits pour appuyer et construire leurs recherches. Dans ce nouvel ordre naissent des cercles de savants qui, vers la fin du XVII ${ }^{\mathrm{e}}$ siècle, se structurent en académies, comme la Royal Society de Londres et l'Académie royale des sciences en France, qui firent paraître les premières revues scientifiques Philosophical Transactions of the Royal Society à Londres et le Journal des savants à Paris. L'Académie des sciences, sous couvert d'une mission d'expertise et de jugement commanditée par la monarchie, prenait le pouvoir dans le domaine de la publication scientifique en s'affranchissant de la censure - et ce faisant de l'autorité de l'Église - et imposait son influence sur les imprimeurs pour s'assurer le contrôle des «médias » de l'époque. Ainsi, une autorité « savante », en se servant de sa qualification d'experts reconnue par l'État, prenait le contrôle des moyens de diffusion et surtout des auteurs, puisque c'est elle qui définissait ce qui relevait ou non du domaine scientifique. À plus d'un titre, comme le souligne Jérome ${ }^{33}$, les manifestations de l'autorité qu'exerçait l'Académie des sciences relèveraient aujourd'hui de l'autoritarisme. Il faut noter que ces publications scientifiques s'adressaient à un vaste public d'érudits avides de connaître les dernières avancées scientifiques et discussions de savants, répondant à la volonté d'élargir le cercle des connaisseurs par des lieux considérés comme des « espaces de rencontre et de débats entre savants et nobles éclairés » ${ }^{34}$, constituant un " dispositif complexe où s'élabore le savoir en même temps qu'il se diffuse $»^{35}$.

Le XVIII ${ }^{\mathrm{e}}$ siècle correspond à ce que Jammes ${ }^{36}$ définit comme "l'apogée du livre scientifique ». L'imprimé devient le vecteur de base de la diffusion du savoir savant, nécessaire pour un savant qui souhaite être reconnu par ses pairs et faire attester la primauté d'une découverte. Comme l'évoque Nicoli ${ }^{37}$, on voit apparaitre le besoin pour l'auteur de " ne pas se borner au pur travail intellectuel : connaître les mécanismes du monde de l'édition et savoir les exploiter devient primordial ». En étudiant la correspondance de représentants des Lumières avec des éditeurs et des libraires, Nicoli a démontré que pour les auteurs, il n'y a pas soumission totale aux académies : il existe des réseaux parallèles « aux marges de la production des savoirs [...] sans le soutien desquels les savants seraient perdus ». Malgré les progrès techniques inhérents à la diffusion de l'information, ce n'est qu'en 1789, avec l'adoption de l'article 11 de la Déclaration des droits de l'homme et du citoyen, proclamant que «La libre communication des pensées et des opinions est un des droits les plus précieux de l'homme » et décrétant « la liberté d'imprimer », que la publication scientifique connut un réel essor du point de vue du rythme

33. Denis Jérome, "L'autorité des grandes revues scientifiques », in Antoine Compagnon (dir.), De l'autorité, Odile Jacob, 2008.

34. Jean Caune, Pour des humanités contemporaines ; science, technique, culture : quelles médiations?, Grenoble, PUG, 2013.

35. Daniel Raichvarg, Sciences pour tous, Paris, Gallimard, 2005.

36. Bruno Jammes, "Le livre de science ", in Roger Chartier \& Henri-Jean Martin, Histoire de l'édition française. Tome 2 : Le livre triomphant, 1660-1830, Paris Fayard, 1990.

37. Miriam Nicoli, "Les coulisses de l'édition scientifique au XVIII ${ }^{\mathrm{e}}$ siècle ", Colloque Histoire des sciences "par en bas ", du 5 au 7 juin 2013, Université du Maine/IUFM, https://www. canal-u.tv/video/universite_du_maine_pole_ressources_numeriques_prn/les_coulisses_de_1_edition_ scientifique_au_xviiie_siecle.14411 
de diffusion et amorça la diversité thématique des revues. Consécutivement, le commerce de l'édition s'organise, comme le démontrent les études menées par Febvre, Chartier, Darnton ou encore Barber ${ }^{38}$. En particulier, la notion d'auteur acquiert un statut juridique et les librairies se voient accorder un «privilège exclusif d'édition », prémices aux maisons d'édition.

La III $^{\mathrm{e}}$ République permet aux universitaires de développer librement leurs travaux de recherche et de nouvelles disciplines commencent à poindre. L'engouement du grand public pour les sciences bat son plein, mais la diffusion des connaissances reste en partie confinée aux salles de cours universitaires, au détriment des éditeurs. Ce n'est qu'à la fin du XIX siècle, face à l'augmentation du volume des publications, que les professions de l'information se structurent et que le "phénomène de la documentation émerge : latent jusque-là, il commence à se démarquer de la bibliothéconomie classique avec le développement de l'information scientifique et technique ${ }^{39}$. L'édition scientifique est également marquée durant le $\mathrm{XX}^{\mathrm{e}}$ par le développement des techniques documentaires. Or, si les professionnels de l'information et de la documentation ne participent pas à proprement parler à la production scientifique, ils contribuent à la diffusion de l'information et des connaissances auprès du public. Malgré les transformations de l'environnement de l'édition scientifique, les Comptes rendus de l'Académie des sciences (CRAS) conservent l'autorité sur la publication des travaux scientifiques durant le $\mathrm{XIX}^{\mathrm{e}}$ et le $\mathrm{XX}^{\mathrm{e}}$ siècle et développent la présentation en séance publique des recherches par leurs auteurs, attestant ainsi de la légitimité et de l'autorité individuelles de l'auteur ${ }^{40}$. Ce bref historique de l'édition scientifique montre que pendant des siècles, l'autorité est restée entre les mains des acteurs des académies et de la société savante agrégeant autour d'eux des professionnels de la diffusion de l'information, qu'il s'agisse de bibliothécaires ou de documentalistes, tous réunis par une vision plus ou moins altruiste de l'édition scientifique.

Les CRAS voient leur notoriété et subséquemment leur autorité décliner dans les années 1970 avec l'arrivée massive de journaux thématiques anglophones, lesquels font évoluer le mode d'évaluation des travaux scientifiques en recourant à un panel d'experts, ce qui a pour effet de réduire considérablement les délais de diffusion. C'est également à cette époque que surgissent des tensions entre les différents acteurs de la production et de l'édition scientifique. Au fur et à mesure que l'État diminue les subventions dédiées à la recherche scientifique, les maisons d'édition deviennent des acteurs incontournables de la diffusion scientifique. Sous le joug de cette nouvelle autorité, l'édition scientifique est précipitée dans la sphère marchande, dont les stigmates se font ressentir dès le début des années 1990 à travers les coûts inflationnistes des revues et des ouvrages circonscrivant l'accès aux institutions et pays riches, l'augmentation des délais de publication et la mise à mal de l'indépendance de l'évaluation ${ }^{41}$.

\section{Ibid.}

39. Jean-Philippe Accart, "Bibliothécaire, documentaliste », Bulletin des bibliothèques de France, 1, 2000, http://bbf.enssib.fr/consulter/bbf-2000-01-0088-011

40. Denis Jérome, "L'autorité des grandes revues scientifiques ", op. cit.

41. Ghislaine Chartron, «Scénarios prospectifs pour l'édition scientifique », Hermès, 57, 2010, p. 123 129. 


\section{DÉVIANCES ET PROFITS DE L'ÉdITION DE LA RECHERCHE}

Force est de constater qu'un déséquilibre de plus en plus prégnant s'est instauré dans la relation entre les éditeurs et les chercheurs. Soulignons que ces derniers étant à la fois les contributeurs et les lecteurs, ils subissent la double peine des éditeurs. Tout d'abord d'un point de vue intellectuel, puisque les chercheurs sont contraints pour progresser dans leur carrière de prendre part de manière active à la diffusion de la recherche, soit de publier des articles (puis de les évaluer), de coordonner des numéros dans des revues scientifiques de préférence notoires dans leur discipline. Revues prestigieuses qui sont - pour la grande majorité d'entre elles détenues par les éditeurs commerciaux. Par ailleurs, certains éditeurs n'hésitent pas à enjoindre les rédacteurs-chercheurs de trouver de nouveaux modèles d'affaire et d'assurer leur promotion pour accroître les ventes et leurs bénéfices. . Corrélée à la première, la seconde peine est financière. Car si depuis la fin des années 1980, les prix pratiqués par les éditeurs n’ont cessé de croître, les rémunérations liées à la cession des droits d'auteur sont à l'inverse de plus en plus minimes, lorsqu'elles subsistent ou qu'elles ne sont pas proposées sous forme de bon d'achat dans la collection de l'éditeur. Ce, alors même qu'une partie des tâches - tapuscrit, mise en page, éléments de couverture, vérification de l'orthographe et des fautes de frappe...-, quand ce n'est pas la totalité, incombe aux auteurs ou coordinateurs et que subséquemment déchargés de ces missions, les éditeurs sont relégués au rang d'imprimeur. Rappelons également que les éditeurs ne rémunèrent pas le comité éditorial, ni les experts. Nous pourrions croire que ceci est dû à la conjoncture actuelle et que le secteur est touché - comme tant d'autres - par la crise. Il n'en est rien, car comme le souligne le rapport EPRIST ${ }^{42}$, le secteur de l'édition scientifique et en particulier de la recherche se porte à merveille ${ }^{43}$. Au regard de ce rapport, les arguments des grands éditeurs évoquant la conjoncture, la profusion de l'offre et la concurrence accrue des autres éditeurs et désormais du libre accès ne tiennent pas. Le raisonnement des éditeurs semble bien plus simple : quel besoin de rémunérer les chercheurs alors qu'ils ont l'injonction institutionnelle de le faire ? Comble du cynisme, ces travaux et suivis intellectuels sont ensuite publiés par l'éditeur et commercialisés auprès de ceux qui les ont produits.

De plus, les acteurs scientifiques ainsi que les professionnels de l'information et de la documentation ne peuvent être que scandalisés de voir que le développement de la publication numérique, qui aurait dû permettre une baisse substantielle des coûts, les a in fine augmentés de manière insensée, rendant l'acquisition des collections de plus en plus difficile pour les institutions. Avec le numérique, les flux documentaires se sont inversés, les bibliothèques ont vu la gestion des collections leur échapper et se sont retrouvées dans l'obligation de les acheter par bouquets aux éditeurs qui gèrent ceux-ci depuis leur serveur ${ }^{44}$. Une étude

42. Michel Vajou, "Résultats financiers 2015 de l'édition scientifique ", RapportEPRISTIntelligence IST, 2016, http://www.eprist.fr/wp-content/uploads/2016/03/I-IST_16_R\%C3\%A9sultats Financiers2015EditionScientifique.pdf.

43. L'édition de la recherche représente $60 \%$ du chiffre d'affaires global et $80 \%$ des profits des grands éditeurs commerciaux.

44. Jean-Michel Salaün, Clément Arsenault, Introduction aux sciences de l'information, La Découverte, 2010 . 
de l'ARL (Association of Research Libraries) montre que sur la période de 1986 à 2006, les coûts relatifs à l'achat de périodiques scientifiques supportés par les bibliothèques américaines ont subi une inflation de $290 \%$. Les « bouquets de périodiques » peuvent désormais atteindre des dizaines voire des centaines de milliers d'euros, ce qui oblige les institutions à scruter à la loupe la consultation de ceux-ci, à reconsidérer leur politique d'acquisition et dans bien des cas à mettre fin à des abonnements. En d'autres termes, les bibliothèques universitaires ne peuvent plus mettre à disposition des acteurs scientifiques la production dont ils sont les auteurs. Depuis 2010, de nombreuses voix s'élèvent pour dénoncer l'autoritarisme économique des éditeurs commerciaux dont les marges annuelles sont spectaculaires ${ }^{45}$ et l'inertie des politiques qui acceptent de payer deux fois le travail des chercheurs ${ }^{46}$. Si les militants du libre accès furent les lanceurs d'alerte, la contestation gagne du terrain tant parmi les professionnels de l'information et des bibliothèques que parmi les chercheurs, dont certains refusent désormais de prendre part au système éditorial commercial. Les récentes difficultés financières rencontrées par les bibliothèques universitaires de Montréal et d'Ottawa ne sont malheureusement pas des cas isolés ${ }^{47}$. Elles les ont simplement publicisés, ce que peu de bibliothèques osent faire.

En d'autres termes, les éditeurs commerciaux profitent pleinement du système de publication, d'évaluation et de « consommation » de la recherche scientifique. Or, parmi les différentes formes de publication liées à l'édition scientifique, les revues scientifiques se sont imposées comme le mode de communication privilégié des chercheurs, d'où les marges confortables liées à l'édition de la recherche pour les grands éditeurs commerciaux. Dans le même temps, associées aux techniques de la bibliométrie, les revues sont devenues de véritables supports pour l'évaluation de la recherche, en témoigne le référentiel de l'AERES ${ }^{48}$ ou le facteur d'impact ${ }^{49}$. Ceci a opéré un basculement, depuis une situation d'observation de l'état de la science à travers la masse des publications pour déterminer de nouveaux axes de recherche vers l'étude de la production d'un chercheur, d'un laboratoire (voire d'un pays) pour l'évolution de carrière ou la dotation de crédits.

45. Michel Vajou, «Résultats financiers 2015 de l'édition scientifique », op. cit.

46. Pierre-Carl Langlais, Rayna Stamboliyska, « La France préfère payer (deux fois) pour les articles de ses chercheurs ", L'Obs Rue89, novembre 2014, http://rue89.nouvelobs. com/2014/11/10/france-prefere-payer-deux-fois-les-articles-chercheurs-255964\#

47. Voir notamment : Joël Lemay, «L’Université de Montréal renonce à 2116 abonnements pour ses bibliothèques ", TVANouvelles, 2016, http://www.tvanouvelles.ca/2016/05/09/luniversite-demontreal-renonce-a-2116-abonnements-pour-ses-bibliotheques; Justine Mercier, "Compressions dénoncées à la bibliothèque de l'Ud'O ", Le Droit, 2016, http://www.lapresse.ca/ le-droit/actualites/education/201610/20/01-5032482-compressions-denoncees-a-la-bibliotheque-deludo.php

48. Revues évaluées par l'AERES (Agence d'évaluation de la recherche et de l'enseignement supérieur) comme tenant une "place importante dans la diffusion des résultats de la recherche à l'échelle nationale et surtout internationale " dans la discipline pour le cas français. http://www.aeresevaluation.fr/Publications/Methodologie-de-l-evaluation/Listes-de-revues-SHS-de-1-AERES.

49. Le facteur d'impact (FI) estime la visibilité d'une revue au regard de la fréquence de citations de ses articles. Le FI entend montrer ainsi les progressions ou rétrogressions de ladite revue ou d'un auteur au sein de la communauté scientifique internationale. 
Mais cette démarche essentielle dans le système actuel est depuis longtemps l'objet de critiques. Ainsi, Jacques ${ }^{50}$ précise qu'il est important de considérer les variations du taux de citation entre les disciplines : dans les mathématiques, l'on peut dénombrer 6 citations par article en moyenne, 14 en chimie, 19 en biomédecine. Par ailleurs, Moles ${ }^{51}$ signale que les États-Unis avaient constaté que le niveau de leurs publications dans le domaine scientifique prenait du retard, surtout par rapport au Japon, car les articles traitaient d'améliorations dans un domaine, mais pas de nouveautés ni de nouveaux champs. Des recherches d'explications ont montré que dans les comités de lecture qui sélectionnaient les articles, les sujets novateurs provoquaient des débats que l'on reportait au dernier jour, puis souvent à une autre cession, d'où des retards d'au moins six mois, voire plus. Le Crosnier ${ }^{52}$ indique dans sa thèse que les bases de données américaines privilégiaient par principe les publications nationales par rapport aux publications des autres pays, et qu'ainsi l'Indian Journal of Malarialogy n'était pas inclus dans le prestigieux Science Citation Index de l'Institute for Scientific Information, avec un facteur d'impact de 0,528 , alors que des revues américaines à 0,001 y figuraient. Pour Gingras ${ }^{53}$, ceci a engendré quelques effets pervers notamment liés au facteur d'impact, lequel mesure la diffusion de la revue et sous-entend que les articles qui y figurent sont de meilleure qualité, alors qu'il « avantage les disciplines rapidement obsolètes (médecine opposée aux sciences sociales) [... et] détourne indirectement de l'étude de sujets locaux, marginaux ou peu à la mode ». Pour Monniaux ${ }^{54}$, l'évaluation bibliométrique des revues scientifiques a contribué à l'« inflation des publications [et aux] exigences des experts de voir citer leurs travaux ou ceux de leurs amis ${ }^{55}$. Ce qui remet également en cause le processus de validation par les pairs des articles (peer review) et conduit Gingras à se poser la question suivante : qui évalue les évaluateurs?

\section{LE NUMÉRIOUE : UN ELdORAdO POUR L'ÉdITION DE LA RECHERCHE ?}

«Ces critiques multipliées et d'autres ont conduit à remettre en cause l'organisation du marché de la publication scientifique dans un contexte où le numérique a ouvert de nouvelles opportunités, de nouvelles potentialités de

50. Jean Jacques, «Débat ", La création scientifique rétribuée, Art et science : de la créativité, colloque de Cerisy 1970, Union générale d'édition, coll. « 12-18», 1972.

51. Abraham Moles, Les sciences de l’imprécis, Seuil, 1998.

52. Hervé Le Crosnier, Systèmes d'accès à des ressources documentaires : vers des anté-serveurs intelligents, thèse en sciences, université Aix Marseille, 1990.

53. Benjamin Caraco, Yves Gingras, «Les dérives de l'évaluation de la recherche. Du bon usage de la bibliométrie. Compte rendu », 2014, https://hal.archives-ouvertes.fr/halshs-01009987/document.

54. David Monniaux, "Pourquoi les universités se désabonnent des revues scientifiques », Ma vérité sur, 2014, http://www.maveritesur.com/david-monniaux/pourquoi-les-universites-se-desabonnentdes-revues-scientifiques/749

55. Peut-être peut-on voir dans ces pratiques une forme de rémunération par reconnaissance... ou plus plausiblement que les experts ne peuvent pas posséder intensité et extensité élevées (Fontanille et Zilberberg) et qu'ils se fient à leur propre construit intellectuel pour évaluer la pertinence des articles. 
diffusion de la recherche. ${ }^{56}$ Las d'un diktat économique de plus en plus souvent au détriment du scientifique, bon nombre de chercheurs se sont rapidement emparés de la Toile pour conduire des projets de publication scientifique et ainsi se soustraire à l'autorité et aux logiques marchandes des grands éditeurs commerciaux. En effet, ces chercheurs ne sont pas restés sourds aux sirènes d'internet, à un espace de liberté d'expression désintermédié ${ }^{57}$ et, de fait, à l'attractivité du libre accès. Avec internet, plus besoin d'imprimeur - ce qui était le seul rôle qui restait à certains éditeurs - et donc la possibilité de contrôler l'ensemble des processus éditoriaux corrélatifs à la diffusion d'une revue scientifique.

Depuis les origines des revues scientifiques numériques, le débat suscité par le libre accès (open access), c'est-à-dire la possibilité d'accéder gratuitement aux publications scientifiques et plus largement aux données de la recherche, enflamme la communauté scientifique ${ }^{58}$. Le mouvement du libre accès est à l'origine de nombreuses initiatives individuelles ou collectives formalisées par des déclarations officielles (Budapest en 2001, Bethesda et Berlin en 2003) et des conventions de recherche. La démocratisation des technologies et subséquemment des pratiques inhérentes à internet ont favorisé la création de « nouveaux espaces intermédiaires [lesquels] s'ajoutent aux expressions médiatiques scientifiques classiques [...] les réseaux de revues sont venus compléter la littérature grise en accès libre, les dépôts institutionnels (pré-prints et post-prints des archives ouvertes) commencent tout juste à s'imposer comme complément plutôt qu'alternative aux revues traditionnelles ${ }^{59}$. Comme le rapporte Guérini, face à cet engouement autour du libre accès, les éditeurs sont de plus en plus obligés d'accepter de nouvelles règles du jeu, en partie par peur de voir les auteurs se détourner définitivement de leur service et, ce faisant, de perdre leurs principales sources de production et de revenu.

Force est néanmoins de constater que la majorité des revues en ligne ne sont qu'une transposition - plus ou moins adaptée au support numérique de leurs homologues imprimées ${ }^{60}$. Toutefois, l'ontophanie numérique ${ }^{61}$, soit l'habituation aux phénomènes numériques, commence à s'instaurer au sein de la communauté scientifique, les " services "Web 2.0" [ont] revitalisé des valeurs comme la participation (discussion), la mutualisation de ressources (collaboration massivement distribuée autour de projets), le partage (fichiers, idées, événements,

56. Ann Shumelda Okerson, James O’Donnell, Scholarly Journals at the Crossroads. A Subversive Proposal for Electronic Publishing, Washington DC, Association of Research Libraries, juin 1995. Cité par Ghislaine Chartron, "Scénarios prospectifs pour l'édition scientifique », op. cit., p. 123-129.

57. Weissberg (1998), cité par Nathalie Pignard-Cheynel, « La publication scientifique sur Internet », in Claude Le Bœuf \& Nicolas Pélissier (dir.), Communiquer l’information scientifique : éthique du journalisme et stratégies des organisations, Paris, L'Harmattan, 2003, p. 367-387.

58. Ghislaine Chartron, "Évolution de l'édition scientifique, 15 ans après », EUTIC 2007, Athènes. 2007.

59. Évelyne Broudoux, Ghislaine Chartron, « La communication scientifique face au Web 2.0. Premiers constats et analyse », H2PTM'09 : Rétrospective et perspective - 1989-2009, Hermès-Lavoisier, 2009, p. 323-336.

60. Lise Verlaet, Sidonie Gallot, Audilio Gonzales Aguilar, «Le paradigme de la complexité. Apports pour les usages formels de l'hypertexte ", in Yves Jeanneret et al. (dir), Pratiques et usages numériques : H2PTM'13, op. cit., p. 75-90.

61. Stéphane Vial, L'être et l'écran : comment le numérique change la perception, Paris, Puf, 2013. 
etc.) $»^{62}$. Au regard des travaux menés à l'heure actuelle, on remarque que les outils d'écrilecture influent certes sur la forme (via de nouveaux architextes), mais surtout sur le fond. En effet, des systèmes de « commentarisation » et d'annotation apparaissent au sein des revues scientifiques. Ces écrits, jusque-là limités à la sphère privée de l'écrilecteur, s'affichent, se partagent, se discutent, ouvrent la voie à l'open science $^{63}$ (science ouverte) et ce faisant à de nouveaux modèles de collaboration et d'évaluation des articles scientifiques.

Parmi les premières initiatives, on peut signaler le serveur de preprints de Los Alamos (arXiv.org) dont le fondateur, Ginsparg, recommande de s'affranchir du système de validation par les pairs et de le remplacer par les outils d'écrilecture avec la possibilité de faire publiquement des commentaires, remarques et corrections sur les articles. Cette mouvance a fait légion outre-Atlantique et l'idée de substituer à l'évaluation par les pairs généralement réalisée en "double aveugle » (double blind) - au sein de laquelle auteur et évaluateurs sont anonymes - une évaluation " ouverte " (open peer reviewing) - auteur et évaluateurs sont connus - gagne du terrain en Europe et inspire de nouveaux modèles de revue scientifique, notamment les "méga-revues " ${ }^{64}$. Les arguments favorables à cette évolution sont multiples, avec en premier lieu la rapidité de diffusion de la recherche à tous les acteurs scientifiques (les jeunes chercheurs sont souvent absents des revues). De plus, selon les partisans de l'évaluation ouverte (open peer reviewing), en fonction des disciplines il est très facile de reconnaître les travaux de tel ou tel chercheur, l'évaluation « à l'aveugle » perd alors toute pertinence. Le processus d'évaluation à l'aveugle est remis en question : ces évaluations sont-elles réellement conduites « à l'aveugle »? Les traces laissées par l'auteur ont-elles bien été effacées (références, propriété du document. ..) ? L'évaluateur a-t-il reconnu ou cherché à reconnaître l'auteur de l'article ? L'autre argument clé de l'évaluation ouverte repose sur l'identification du rapporteur de l'article. Le fait que le rapporteur soit identifié et identifiable constituerait un rempart contre les évaluations trop laconiques ou trop virulentes, un gage d'évaluateurs intéressés par le contenu scientifique exposé, cette tâche ne leur étant pas imposée, pouvant subséquemment conduire à un débat avec les auteurs et à de nouvelles collaborations. On s'orienterait alors vers une autorité collective et collaborative de la publication scientifique. Toutefois, pour que ce système d'évaluation ouverte et transparente puisse non seulement s'imposer mais être pérenne, il est indispensable que les acteurs scientifiques acquiescent et

62. Évelyne Broudoux, Ghislaine Chartron, «La communication scientifique face au Web 2.0 », op. cit. 63. "Le concept de base de l'open science est de permettre, comme pour le logiciel libre, un accès universel au progrès de la connaissance, en organisant une collaboration mondiale entre les chercheurs à partir des nouveaux outils de communication. Y parvenir suppose de mettre en place un système permettant le partage des données, de la méthodologie, des outils (notamment les logiciels), pour permettre à chaque chercheur de contribuer facilement au progrès de la connaissance commune. Ainsi, les résultats de chacun enrichissent la recherche collective » (Corine Lepage, "L'open science, un dé pour les États ", Cahiers Droit, Sciences \& Technologies, 3, 2010, p. 21-29).

64. Voir notamment : Bo-Christer Björk, "A study of innovative features in scholarly open access journals", Journal of Medical Internet Research, 13(4), 2011, http://www.jmir.org/2011/4/e115/; Catriona J. MacCallum, "Why ONE Is More Than 5", PLoS Biology, 9(12), 2011, https://doi.org/10.1371/journal.pbio.1001235; Maria Cassela, "Innovation and experimentation in scholarly publishing: Web 2.0 features in Open Access journals", IFLA, Helsinki, 2012, http://conference.ifla.org/past-wlic/2012/149-cassella-en.pdf. 
prennent part à cette nouvelle logique de jeu. Nous n'avons pas le recul suffisant pour analyser ces nouveaux modes d'évaluation, mais les principaux risques que nous voyons sont les suivants : d'une part - n'ayant plus l'obligation d'évaluer les articles, ce qui demande un travail important et les articles étant néanmoins publiés - il n'y aurait plus d'évaluation, ce qui entraînerait potentiellement une chute de la qualité scientifique des publications. D'autre part, le principe de transparence des évaluateurs pourrait conduire à un consensus mou dans la validation scientifique. Conscients des risques relatifs à une évaluation uniquement ouverte des articles, d'autres créateurs de revues scientifiques prônent le maintien du système d'évaluation par les pairs, dont la fonction serait toujours de faire une première sélection des articles, tout en proposant dans un second temps un système de «commentaires ouverts " (open peer commentary), lequel peut, selon les cas, être limité à la phase d'évaluation ou permanent ${ }^{65}$. Les articles sont publiés avec l'intégralité des commentaires voire des débats qui ont suivi avec l'auteur ${ }^{66}$.

Quelle que soit la politique éditoriale choisie - procédures d'évaluations ouvertes ou hybrides -, il n'en reste pas moins que l'utilisation des dispositifs numériques est en train de redistribuer les cartes de l'autorité scientifique, donnant ainsi du jeu à ceux qui font la science. Il n'y a qu'un pas du libre accès (open access) à la science ouverte (open science), et la perspective d'une autorité collective et collaborative n'est plus seulement idéelle mais pourrait se concrétiser si les chercheurs exploitent correctement leur jeu. Mais si l'autorité devient collective et collaborative, sur qui ou sur quoi repose l'autorité scientifique ? La mise en place d'outils d'écrilecture au sein des revues scientifiques autorise les lecteurs non plus seulement à accéder aux contenus, mais également à les commenter, les évaluer, les modifier, les corriger. En ce sens, les fonctions « lecture-écriture » s'entremêlent, voient leurs prescriptions augmentées et, de fait, les rôles du chercheur se démultiplier. À ce titre, Lefebvre ${ }^{67}$ distingue le chercheur-auteur, le chercheur-rapporteur, le chercheur-lecteur et le chercheur-commentateur. Nos recherches ${ }^{68}$ caractérisent un nouveau type de chercheur, le chercheur-baliseur, dont la fonction est de faire émerger l'univers conceptuel des articles tel que relaté par les auteurs. Comme mis en ouvre au sein de la revue $\operatorname{COSSI}^{69}$, ceci conduit à formaliser un nouveau mode d'accès aux contenus via des « fiches concepts » et doit permettre in fine de co-construire une ontologie. Cette ontologie - nécessairement incomplète et évolutive - est présentée sous une forme graphique aux lecteurs ${ }^{70}$.

65. Voir notamment: Nathalie Pignard-Cheynel, « La publication scientifique sur Internet », in Claude Le Bœuf \& Nicolas Pélissier (dir.), Communiquer l'information scientifique : éthique du journalisme et stratégies des organisations, op. cit., p. 367-387; Hans Dillaerts, Libre accès à la communication scientifique et contexte français : développement et enjeux pour la créativité et l'interdisciplinarité, thèse de doctorat, CNAM, 2012, [en ligne] https://tel.archives-ouvertes.fr/tel-00768432.

66. Julien Bordier, «Évaluation ouverte par les pairs : de l'expérimentation à la modélisation : Récit d'une expérience d'évaluation ouverte par les pairs ", 2016, <hal-01283582>.

67. Muriel Lefebvre, "Rendre public le processus d'évaluation de la recherche », Réseaux, 6, 2010, p. 71-96.

68. Lise Verlaet, La recherche pertinente sur le Web. Concevoir un dispositif d'information adapté aux activités cognitives des lecteurs, Sarrbrücken, Éditions Universités Européennes, 2011.

69. Revue Communication, Organisation, Société du Savoir et Information, http://www.revue-cossi.info/.

70. Intégration des information-visualisation est prévue en mai 2017. 
L'interaction entre les concepts, à l'instar des interactions entre acteurs ${ }^{71}$, est plus qu'un simple lien entre deux données, plus que des informations, elle permet la construction de nouveaux schèmes dans l'esprit des lecteurs et de générer de nouvelles significations, qui peuvent conduire à de nouvelles découvertes notamment par l'info-visualisation. Le chercheur-baliseur a la responsabilité de libérer la mémoire encyclopédique, de la soumettre à la critique des lecteurs, de permettre aux lecteurs d'être proactifs dans la co-construction de l'ontologie de leur domaine. Ainsi, cet outil d'écrilecture, qui repose sur l'autorité collective et collaborative des chercheurs-auteurs-baliseurs, entend repositionner au centre du débat scientifique les concepts qui fondent la science et subséquemment co-construire l'autorité ontologique, à travers ce que l'on pourrait qualifier d' « altruisme scientifique ».

\section{Conclusion}

Comme nous l'avons énoncé, la révolution gutenbergienne a constitué un tournant important pour la science en permettant à ses acteurs d'interagir et d'avoir accès à un grand nombre d'informations. Et s'il est indéniable que des pratiques de production ont préexisté à l'imprimerie, celles-ci ont pu prendre une tout autre ampleur notamment par l'accélération des communications scientifiques et subséquemment des débats d'idées. Toutefois, pour acquérir de l'autorité - laquelle réside dans la notoriété de l'auteur et est mesurée par le nombre de citations des travaux, le nombre d'exemplaires imprimés -, les savants ont dû faire face à la censure de l'Église puis - après l'intervention de la monarchie - à l'autorité des académies garantes de la qualité scientifique des écrits, évalués au regard des paradigmes défendus par ses représentants. À ce titre, «la liberté d'imprimer » a créé un véritable appel d'air tant au regard du rythme de diffusion qu'à celui de la pluralité des points de vue. La quantité ne fait néanmoins pas la qualité, la citation en soi ne confirme ni n'infirme les travaux d'un auteur, elle marque juste le fait que ledit travail a été lu. Pour qu'il soit lu, il est indispensable que celui-ci ait été correctement diffusé. Avec le retrait des financements étatiques, c'est en grande partie sur l'éditeur que repose cette fonction de promotion et de commercialisation des recherches scientifiques. La tâche devenant plus aisée dès lors que l'auctorialité est avérée. Ainsi, les éditeurs commerciaux sont peu à peu devenus des acteurs clés dans le système de la recherche scientifique. Mais l'absence d'une autorité régulatrice dans la relation éditeur-auteur a conduit ces premiers - pour une partie d'entre eux - à profiter du système d'évaluation de la recherche, soit de la nécessité pour ces derniers de publier leurs travaux pour exister au sein de leur discipline et progresser dans leur carrière. À l'heure actuelle, et comme nous l'avons souligné, le déséquilibre est tel dans l'édition de la recherche qu'il n'est pas choquant dans certains cas de parler de « détournement de fonds publics ${ }^{72}$.

71. Karl E. Weick, Sensemaking in organization, Thousand Oaks, CA, Sage Publications, 1995.

72. Jérôme Valluy, "Le libre accès aux publications de SHS, entre marché et État : comment articuler édition numérique en libre accès et... liberté(s) intellectuelles des auteurs \& lecteurs ? ", Séminaire NumeRev, MSH-Sud/université Paul-Valéry, Montpellier, 28 février 2017, http://numerev.com/les-seminaires/16-le-libre-acces-aux-publications-de-shs-entre-marche-et-etatcomment-articuler-edition-numerique-en-libre-acces-et-liberte-s-intellectuelles-des-auteurs-lecteurs. 
La révolution numérique n'est pas étrangère à cette prise de conscience. En à peine deux décennies, internet et le numérique ont modifié les pratiques et les usages liés à l'édition de la recherche, impactant directement le secteur de l'édition (éditeurs, diffuseurs, libraires) en faisant voler en éclat sa principale valeur ajoutée, soit sa capacité de produire, promouvoir et vendre du « papier ». Désormais, il est possible pour un scientifique de diffuser en libre accès ses travaux de recherche, ce, sans grande connaissance technique des dispositifs numériques. Ces nouvelles modalités de diffusion et de gestion de format ont inévitablement eu des répercussions sur l'ensemble des professions de l'information - y compris celles de la documentation et des bibliothèques -, lesquelles doivent s'adapter aux incessantes innovations technologiques et à l'obsolescence rapide des dispositifs mis en place. Le numérique revisite également le rapport des chercheurs à l'information et à la communication, favorisant par là même les dispositifs d'écrilecture, les pratiques collaboratives et in fine ouvrant la voie à la science ouverte, à une autorité scientifique collaborative qui par nature s'intéresserait aux construits communs et pourrait conduire à une autorité ontologique.

Cela dit, il ne faut pas croire que l'eldorado supposé du numérique soit sans écueil pour la recherche scientifique. L'héritage des pratiques antérieures contribue autant qu'il limite le développement de pratiques propres au numérique. Ainsi, le facteur d'impact ne sera pas un lointain souvenir, il a d'ores et déjà été suppléé par les altmetrics ${ }^{73}$, les traitements bibliométriques à l'instar d'autres pratiques se transforment et s'adaptent aux nouveaux progrès techniques. Le numérique est porteur tout à la fois d'utopies et de dystopies car le système documentaire que représente internet permet autant le partage de connaissances, l'accès et la démocratisation des savoirs, la tolérance, que la surveillance généralisée et le totalitarisme ${ }^{74}$. Les pouvoirs politiques semblent dépassés par le phénomène numérique, peinent à légiférer et à instaurer des autorités de régulation. Soulignons néanmoins la récente promulgation de la « Loi pour une république numérique » 75 qui devrait venir rééquilibrer la relation éditeur-auteur en ce qui concerne l'édition de la recherche.

73. Nathalie Clairoux, «Altmetrics : des indicateurs d'impact immédiat », Université de Montréal, 2016, http://fr.slideshare.net/nclairoux/altmetrics-une-introduction-pour-les-chercheurs.

74. Voir notamment : Thierry Libaert, La transparence en trompe-l'œil, Descartes \& Cie, 2003 ; Sidonie Gallot, Lise Verlaet, "La transparence : l'utopie du numérique », Communication \& Organisation, 49, 2016, p. 203-217.

75. Publiée au Journal Officiel du 8 octobre 2016. « Libre accès aux résultats des travaux de recherche publique et autorisation de la fouille de textes et de données. Les résultats de travaux de recherche financés à plus de $50 \%$ par des fonds publics pourront être mis en ligne en libre accès par leurs auteurs, après une période d'embargo de 6 à 12 mois. Cette mesure facilitera la libre diffusion de résultats de recherche dont la diffusion était auparavant restreinte et rendue payante par les éditeurs. Le projet de loi autorise également la fouille de textes et de données en ligne, une pratique essentielle dans le cadre de recherches en sciences humaines et sociales, qui était jusqu'à présent restreinte par les droits de propriété intellectuelle », http://www.gouvernement.fr/action/pour-une-republique-numerique. 


\section{Références}

Accart Jean-Philippe, «Bibliothécaire, documentaliste », Bulletin des bibliothèques de France, 1, 2000, [en ligne] http://bbf.enssib.fr/consulter/bbf-2000-01-0088-011

Beaudry Guylaine, La communication scientifique et le numérique, Paris, Hermès Lavoisier, 2011

Björk Bo-Christer, "A study of innovative features in scholarly open access journals ", Journal of Medical Internet Research, 13(4), 2011, [en ligne] http://www.jmir.org/2011/ $4 / \mathrm{e} 115 /$

Bordier Julien, « Évaluation ouverte par les pairs : de l'expérimentation à la modélisation : Récit d'une expérience d'évaluation ouverte par les pairs » 2016, <hal-01283582>

Bourricaud François, Esquisse d'une théorie de l'autorité, Paris, Plon, 1969

Broudoux Évelyne, "Contours du document numérique connecté », Documents et dispositifs à l'ère post-numérique : CIDE18, Éditions Europia, 2015, p. 7-16

Broudoux Évelyne, "L'écosystème scientifique à l'heure de la participation sur le Web ", in Yves Jeanneret et al. (dir.), Pratiques et usages numériques: H2PTM'13, Hermès-Lavoisier, 2013, p. 239-256

Broudoux Évelyne, Chartron Ghislaine, "La communication scientifique face au Web2.0. Premiers constats et analyse ", H2PTM'09 : Rétrospective et perspective - 1989-2009, Hermès-Lavoisier, 2009

Cassela Maria, "Innovation and experimentation in scholarly publishing: Web 2.0 features in Open Access journals », IFLA, Helsinki, 2012, [en ligne] http://conference. ifla.org/past-wlic/2012/149-cassella-en.pdf

Caraco Benjamin, Gingras Yves, "Les dérives de l'évaluation de la recherche. Du bon usage de la bibliométrie. Compte rendu », 2014, https://hal.archives-ouvertes.fr/halshs01009987/document

Caune Jean, Pour des humanités contemporaines ; science, technique, culture : quelles médiations ?, PUG, 2013

Chante Alain, «La notion de catalogue : de l'imprimé au numérique », Culture \& musées, 21, 2013, p. 131-152

Chartron Ghislaine, "Évolution de l'édition scientifique, 15 ans après ", EUTIC 2007, Athènes, 2007, <sic_00186675>

Chartron Ghislaine, «Scénarios prospectifs pour l'édition scientifique », Hermès, 57, 2010, p. 123-129

Chartron Ghislaine, "Tendances lourdes et tensions pour les filières du document numérique ", Le "Document » à l'ère de la différenciation numérique, Europia, 2012, https://hal.archives-ouvertes.fr/sic_00634783/document

Clairoux Nathalie, "Altmetrics: des indicateurs d'impact immédiat », université de Montréal, 2016, [en ligne] http://fr.slideshare.net/nclairoux/altmetrics-une-introduction-pour-leschercheurs

Clément Jean, « Du livre au texte Les implications intellectuelles de l'édition électronique », Revue Sciences et techniques éducatives, 5(4), 1998, p. 401-409, <edutice-00135808>

Compagnon Antoine, "Qu'est-ce qu'un auteur ? Introduction : mort et résurrection de l'auteur ", cours de licence, université Paris-Sorbonne, http://www. fabula.org/compagnon/auteur1.php

Couzinet Viviane, «Fabrique de la liste : un dispositif entre mémoire et commémoration », $2^{\mathrm{a}}$ Jordana Cientifica Internacional Redes e Processos Info-Communicacionais : Mediaçiões, Memoras, Apropriações, Rio de Janeiro, 2012, p 132-151 
Delsol Chantal, L'autorité, Paris, Puf, 1994

Dillaerts Hans, Libre accès à la communication scientifique et contexte français : développement et enjeux pour la créativité et l'interdisciplinarité, thèse de doctorat, CNAM, 2012, https://tel.archives-ouvertes.fr/tel-00768432

Foucault Michel, «Qu'est-ce qu'un auteur ?», 1969, Dits et écrits, Gallimard, 1994

Foucault Michel, Les mots et les choses, Gallimard, 1966

Fraysse Patrick, Régimbeau Gérard, «Le patrimoine architectural entre monuments-phares et documents monumentaires ", La transmission des connaissances, des savoirs et des cultures : Alexandrie, métaphore de la francophonie, Actes du Troisième colloque international du CIDEF (Centre international de documentation et d'échanges de la francophonie - Québec) - AFI (Agora francophone internationale - Paris), Alexandrie (Égypte), Bibliothèque d'Alexandrie, du 12 au 15 mars 2006, http://hal.archivesouvertes.fr/hal-00607126/ [12 p.]

Fraysse Patrick, Le patrimoine monumental en images : des méditations informationnelles à la conversion monumentaire des documents, thèse de doctorat en SIC, université Toulouse 2, 2006

Gallot Sidonie, Verlaet Lise, «La transparence : l'utopie du numérique », Communication \& Organisation, 49, 2016, p. 203-217

Garapon Antoine, Perdriolle Sylvie, Quelle autorité ? : une figure à géométrie variable, Autrement, 2000

Houssaye Jean, Autorité ou éducation : Entre savoir et socialisation : le sens de l'éducation, ESF, 2007

Jacques Jean, "Débat ", La création scientifique rétribuée, Art et science : de la créativité, colloque de Cerisy 1970, Union générale d'édition, 1972

Jammes Bruno, «Le livre de science », in Roger Chartier \& Henri-Jean Martin (dir.), Histoire de l'édition française. Tome 2 : Le livre triomphant, 1660-1830, Paris Fayard, 1990

Jérome Denis, «L'autorité des grandes revues scientifiques », in Antoine Compagnon (dir.), De l'autorité, Paris, Odile Jacob, 2008

Juignet Patrick, "Michel Foucault et le concept d'épistémè », Philosophie, science et société. 2015, http://www.philosciences.com/Pss/philosophie-generale/la-philosophie-etsa-critique/10-michel-foucault-episteme

Karila-Cohen Pierre, "L'autorité, objet d'histoire sociale », Le Mouvement Social, 3, 2008, p. 3-8

Kojève Alexandre, La notion de l'autorité [1942], Paris, Gallimard, 2004

Langlais Pierre-Carl, Stamboliyska Rayna, " La France préfere payer (deux fois) pour les articles de ses chercheurs ", L'Obs Rue89, novembre 2014, http://rue89. nouvelobs.com/2014/11/10/france-prefere-payer-deux-fois-les-articles-chercheurs-2559 64\#

Le Crosnier Hervé, Systèmes d'accès à des ressources documentaires : vers des anté-serveurs intelligents, thèse en sciences, université Aix Marseille, 1990

Lemay Joël, " L’Université de Montréal renonce à 2116 abonnements pour ses bibliothèques ", TVANouvelles, 2016, http://www.tvanouvelles.ca/2016/05/09/luniversite -de-montreal-renonce-a-2116-abonnements-pour-ses-bibliotheques

Lepage Corine, «L'open science, un dé pour les États », Cahiers Droit, Sciences \& Technologies, 3 | 2010, p. 21-29

MacCallum Catriona J., "Why ONE Is More Than 5 ", PLoS Biology, 9(12), 2011, https://doi.org/10.1371/journal.pbio.1001235

Mendes Gallinari Melliandro, "La "clause auteur" : l'écrivain, l'ethos et le discours littéraire », Argumentation et Analyse du Discours, 3, 2009, http://aad.revues. org/663 
Mercier Justine, "Compressions dénoncées à la bibliothèque de l'Ud'O ", Le Droit, 2016, http://www.lapresse.ca/le-droit/actualites/education/201610/20/01-5032482compressions-denoncees-a-la-bibliotheque-de-ludo.php

Moles Abraham, Les sciences de l'imprécis, Seuil, 1998

Monniaux David. "Pourquoi les universités se désabonnent des revues scientifiques ", Ma vérité sur, 2014, http://www.maveritesur.com/david-monniaux/pourquoi-lesuniversites-se-desabonnent-des-revues-scientifiques/749

Nicoli Miriam, "Les coulisses de l'édition scientifique au XVIII ${ }^{\mathrm{e}}$ siècle ", Colloque Histoire des sciences "par en bas ", du 5 au 7 juin 2013, université du Maine / IUFM, https://www.canal-u.tv/video/universite_du_maine_pole_ressources_numeriques_prn/ les_coulisses_de_1_edition_scientifique_au_xviiie_siecle.14411

Okerson Ann Shumelda, O'Donnell James, Scholarly Journals at the Crossroads. A Subversive Proposal for Electronic Publishing, Washington DC, Association of Research Libraries, 1995

Pignard-Cheynel Nathalie, «L'édition de revues scientifiques. Une forme de marchandisation de la diffusion de connaissances », Sciences de la Société, 66, 2005, p. 181-198

Pignard-Cheynel Nathalie, «La publication scientifique sur Internet », in Claude Le Bøuf \& Nicolas Pélissier (dir.), Communiquer l'information scientifique : éthique du journalisme et stratégies des organisations, Paris, L'Harmattan, 2003, p. 367-387

Pontille David, «La signature scientifique. Authentification et valeur marchande » Actes de la Recherche en Sciences sociales, 2002, p. 141-142

Raichvarg Daniel, Sciences pour tous, Paris, Gallimard, 2005

Revault d'Allonnes Myriam, Le pouvoir des commencements. Essai sur l'autorité, Paris, Seuil, 2006

Rieffel Rémy, Révolution numérique, révolution culturelle?, Paris, Gallimard, 2014

Salaün Jean-Michel, Arsenault Clément, Introduction aux sciences de l'information, Paris, La Découverte, 2010

Sennett Richard, Autorité, Paris, Fayard, 1980

Souchier Emmanuël, «L'écrit d'écran, pratiques d'écriture \& informatique », Communication \& langages, 107, 1996, p. 105-119

Touitou Cécile, "Les nouveaux usages des générations internet ", $B B F, 4$, juillet 2008, p. 67-70, <http://bbf.enssib.fr/consulter/bbf-2008-04-0067-001>

Vajou Michel, "Résultats financiers 2015 de l'édition scientifique " Rapport EPRIST Intelligence IST, 2016, http://www.eprist.fr/wp-content/uploads/2016/03/I-IST_16_R\% C3\%A9sultatsFinanciers2015EditionScientifique.pdf

Valluy Jérôme, "Le libre accès aux publications de SHS, entre marché et État : comment articuler édition numérique en libre accès et... liberté(s) intellectuelles des auteurs \& lecteurs ? ", Séminaire NumeRev, MSH-Sud / université Paul-Valéry, Montpellier, 28 février 2017, http://numerev.com/les-seminaires/16-le-libre-acces-aux-publicationsde-shs-entre-marche-et-etat-comment-articuler-edition-numerique-en-libre-acces-etliberte-s-intellectuelles-des-auteurs-lecteurs

Verlaet Lise, La recherche pertinente sur le Web. Concevoir un dispositif d'information adapté aux activités cognitives des lecteurs, Sarrbrücken, Éditions Universités Européennes, 2011

Verlaet Lise, Gallot Sidonie, Gonzales Aguilar Audilio, « Le paradigme de la complexité. Apports pour les usages formels de l'hypertexte », in Yves Jeanneret et al. (dir), Pratiques et usages numériques : H2PTM'13, Hermès-Lavoisier, 2013, p. 75-90

Vial Stéphane, L'être et l'écran : comment le numérique change la perception, Paris, Puf, 2013

Vielliard Françoise, "Auteur et autorité dans la littérature occitane non lyrique », Auctor et auctoritas: Invention et conformisme dans l'écriture médiévale, École des Chartes, 2001, p. $375-389$ 
Vuillemin Alain, "La lecture interactive et l'écrilecture ", in Alain Vuillemin \& Michel Lenoble (dir.), Littérature, informatique, lecture, Limoges, Presses universitaires de Limoges, 1999, p. 101-110

Weber Max, Économie et société [1922], Paris, Pocket, 2003

Weick Karl E., Sensemaking in organization, Thousand Oaks, CA, Sage Publications, 1995

Willett Gilles (dir.), La communication modélisée, Ottawa, Édition Erpi, 1992

\section{LISE VERLAET, ALAIN CHANTE \\ lise.verlaet@univ-montp3.fr \\ alain.chante@univ-montp3.fr}

